\title{
1 KMT5C displays robust retention and liquid-like behavior in phase separated
}

\section{2 heterochromatin}

4 Hilmar Strickfaden, ${ }^{1}$ Kristal Missiaen, ${ }^{1}$ Michael J. Hendzel, ${ }^{1,2}$ and D. Alan Underhill1 ${ }^{1,3, *}$

5 Departments of ${ }^{1}$ Oncology, ${ }^{2}$ Cell Biology, and ${ }^{3}$ Medical Genetics, Faculty of Medicine \&

6 Dentistry, University of Alberta, Edmonton, Alberta, Canada

8 Abstract

9 The pericentromere exists as a distinct chromatin compartment that is thought to form by a

10 process of phase separation. This reflects the ability of the heterochromatin protein CBX5 (aka

$11 \mathrm{HP}(\alpha)$ to form liquid condensates that encapsulate pericentromeres. ${ }^{1,2}$ In general, phase

12 separation compartmentalizes specific activities within the cell, but unlike membrane-bound

13 organelles, their contents rapidly exchange with their surroundings. ${ }^{3}$ Here, we describe a novel

14 state for the lysine methyltransferase KMT5C where it diffuses within condensates of

15 pericentromeric heterochromatin but undergoes strikingly limited nucleoplasmic exchange,

16 revealing a barrier to exit similar to that of biological membranes. This liquid-like behavior maps

17 to a discrete protein segment with a small number of conserved sequence features and containing

18 separable determinants for localization and retention that cooperate to confer strict spatial

19 control. Accordingly, loss of KMT5C retention led to aberrant spreading of its catalytic product

20 (H4K20me3) throughout the nucleus. We further found that KMT5C retention was reversible in

21 response to chromatin state, which differed markedly for CBX5 and the methyl-CpG binding

22 protein $\mathrm{MeCP}$, revealing considerable plasticity in the control of these phase separated

23 assemblies. Our results establish that KMT5C represents a precedent in the biological phase 
24 separation $^{4}$ continuum that confers robust spatial constraint of a protein and its catalytic activity

25 without progression to a gel or solid.

Analyses of the prototypic heterochromatin protein CBX5 indicated that phase separation

27 plays an important role in partitioning of pericentromeric heterochromatin. ${ }^{1,2}$ This is particularly

28 evident in mouse interphase nuclei where pericentromeres from multiple chromosomes form

29 chromocenters. ${ }^{5}$ Despite the stable appearance of such assemblies, they are highly dynamic and

30 their protein constituents rapidly exchange with their surroundings, reflecting low affinity,

31 multivalent interactions that allow proteins to coalesce into distinct aqueous compartments. ${ }^{6,7}$ In

32 this context, CBX5 enrichment within pericentromeric heterochromatin requires binding to

33 trimethyllysine-9 on histone $\mathrm{H} 3$ (H3K9me3), which is placed by the lysine methyltransferases

$34 \mathrm{SUV} 39 \mathrm{H} 1$ and $2 .{ }^{8} \mathrm{CBX} 5$ then recruits KMT5C protein to catalyze histone $\mathrm{H} 4$ lysine-20

35 trimethylation (H4K20me3). In fluorescence recovery after photobleaching (FRAP), CBX5

36 rapidly exchanged between the pericentromere and nucleoplasm, ${ }^{9}$ whereas SUV39H2 and

37 KMT5C appeared to be immobile when entire chromocenters were bleached. ${ }^{10}$ Although

38 concluding that the latter two proteins created a stable scaffold, ${ }^{10}$ these studies did not assess

39 mobility within chromocenters, which would instead suggest retention within a liquid

40 compartment. To test this fundamentally different model, we queried KMT5C dynamics within

41 mouse chromocenters in comparison to $\mathrm{CBX} 5$ and $\mathrm{MeCP} 2$, which also exhibits pericentromeric

42 enrichment and nucleoplasmic exchange. ${ }^{10,11}$ As previously shown with full chromocenter

43 bleaching, ${ }^{10,12}$ KMT5C displayed long recovery times, indicating minimal exchange with the

44 surrounding nucleoplasm, and was considerably slower than either CBX5 or MeCP2 (Fig. 1a,

45 Extended Data Videos 1-3). Upon partial bleaching, however, KMT5C fluorescence recovered

46 (Fig. 1a, Extended Data Videos 4 and 5) and progressed from the non-bleached portion of the 
47 chromocenter (Fig. 1b). This occurred on a timescale where there was no appreciable recovery of

48 fully bleached chromocenters in the same nucleus (Fig. 1b), establishing that KMT5C moved

49 readily within chromocenters but did not efficiently exchange (Fig. 1c). In the context of phase

50 separation, this signifies a remarkable barrier to exit that has not been observed for other proteins

51 without transition to a solid or gel state, which renders them immobile. ${ }^{13}$ KMT5C therefore

52 demonstrates that phase separation can achieve robust compartmentalization while nevertheless

53 retaining liquid-like mobility.

Consistent with this behavior, KMT5C was more efficient in chromocenter localization

55 than CBX5 (Fig. 1d, e). Unexpectedly, this enrichment did not simply reflect retention because

56 the unrelated MeCP2 protein had a high partition coefficient (Fig. 1d, e) but underwent

57 nucleoplasmic exchange (Fig. 1a). MeCP2 therefore defined a third state with regard to

58 partitioning and mobility, indicating these parameters were separable and occur across a

59 continuum. Intrinsically disordered regions are also typical of phase-separated proteins, ${ }^{13,14}$

60 including CBX5. ${ }^{1,2}$ Although these features were highly conserved in disorder plots of CBX5

61 and MeCP2 from representative mammals, they varied for KMT5C along with charge properties

62 (Fig. 2a, Extended Data Table 1). Whereas MeCP2 had the highest overall percentage of disorder

63 (76.7), KMT5C exhibited the lowest (33.6), lacked extended regions of disorder, and its profile

64 was most divergent (Fig. 2a, Extended Data Table 1). This suggests that while CBX5 and

$65 \mathrm{MeCP} 2$ have prototypic features of phase separating proteins, KMT5C achieves demixing

66 through other means. To this end, CBX5 and MeCP2 contain highly conserved domains that

67 confer localization to constitutive heterochromatin (Fig. 2a), ${ }^{15-17}$ which for KMT5C involves a

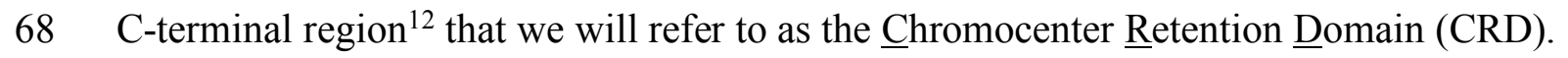

69 Sequence identity within the CRD, however, was limited to 18 of 59 residues across mammals 
70 (Fig. 2b) and its disorder potential and charge properties (Fig. 2c, Extended Data Table 1) varied

71 considerably (Fig. 2d). To broadly establish functional relevance of the CRD, we assessed

72 versions from Homo sapiens $(\mathrm{Hs})$ and Mus musculus $(\mathrm{Mm})$, together with Cavia porcellus $(\mathrm{Cp})$

73 and Bubalus bubalis $(\mathrm{Bb})$ because they exhibited distinct disorder profiles and the greatest range

74 in pI (8.94-11.92) (Fig. 2c, d). Nevertheless, each CRD derivative displayed robust chromocenter

75 partitioning (Fig. 2e, f) that was not significantly different from full-length KMT5C, together

76 with intra-chromocenter mobility and limited exchange (Fig. 2g, Extended Data Videos 6-9). As

77 a result, the dynamic properties of full-length KMT5C can be entirely recapitulated by this short

78 protein segment. Although best exemplified by the mouse CRD, the behavior was shared by all

79 orthologs, suggesting it is driven by common sequence features that are modulated by species-

80 specific differences.

81 We next examined the role of conserved residues in the $\mathrm{CRD}$, focusing on $\mathrm{C}^{362} \mathrm{C}^{366}$,

$82 \quad \mathrm{H}^{357} \mathrm{H}^{365}$, and $\mathrm{W}^{359} \mathrm{~W}^{390} \mathrm{~W}^{392} \mathrm{Y}^{396}$ (Fig. 2b), because they resembled features found in chromatin

83 reader modules that jointly recognize DNA and histones. ${ }^{18}$ Mutation of individual motifs caused

84 elevated exchange in FRAP assays (Fig. 3a, Extended Data Videos 10-12), which was

85 accompanied by increased nucleoplasmic abundance (Fig. 3b) and decreased partitioning (Fig.

$863 \mathrm{c}, \mathrm{d})$. Combining mutants $\left(\mathrm{C}^{366} \mathrm{~W}^{390} \mathrm{~W}^{392}\right)$ abrogated chromocenter localization and led to rapid

87 mobility (Fig. 3a-d, Extended Data Video 13). These findings established that the CRD has

88 evolved multiple determinants that cooperate to confer localization and limit its exit from

89 individual chromocenters, while still supporting a liquid-like state. Non-membranous organelles

90 that form by phase separation are typically spherical, reflecting a reduction in their surface

91 area, ${ }^{19}$ which prompted us to evaluate this parameter in CRD mutants (Fig. 3e). Importantly,

92 each of the mutants caused a reduction in sphericity that correlated with their partitioning and 
93 dynamic behavior (Fig. 3a-d), with $\mathrm{C}^{362} \mathrm{C}^{366}$ being most severe and $\mathrm{W}^{359} \mathrm{~W}^{390} \mathrm{~W}^{392} \mathrm{Y}^{396}$ the least.

94 The extent mutants perturbed CRD activity therefore reflected the degree to which they reduced

95 the valency or interaction affinity, a finding that is consistent with the behavior of other phase

96 separated proteins. ${ }^{13}$ We extended this analysis to include KMT5C, CBX5, MeCP2, and the

97 remaining CRDs. Strikingly, KMT5C supported a significantly higher degree of sphericity than

98 either CBX5 or MeCP2 (Extended Data Fig. 1a), indicating it was more effective at generating

99 surface tension at the boundary between the chromocenter and nucleoplasm. Moreover, all CRDs

100 shared this property (Extended Data Fig. 1b), further highlighting that this domain is the key

101 determinant of the biophysical characteristics of KMT5C.

Phase separation by CBX5 is abrogated in Suv39h1/2 null cells or by mutation that

103 disrupts H3K9me3 recognition, ${ }^{1}$ underscoring the importance of this interaction in seeding liquid

104 demixing. We found the dynamic behavior of KMT5C exhibited the same dependency, reflected

105 by its increased mobility and dispersal in cells lacking SUV39H1/2 (Fig. 4a, Extended Data

106 Videos 14, 15). MECP2, however, displayed more efficient localization and slightly reduced

107 mobility (Extended Data Fig. 2), indicating the chromocenter can support distinct phase

108 separated assemblies depending on chromatin context. Another facet of phase separation

109 involves its reversibility in response to cellular queues. ${ }^{13}$ To this end, we evaluated the histone

110 deacetylase inhibitor Trichostatin A (TSA) because it was known to cause CBX5 displacement

111 from pericentromeric heterochromatin and increase its mobility. ${ }^{20} \mathrm{KMT} 5 \mathrm{C}$, however, largely

112 retained chromocenter localization, but with elevated nucleoplasmic exchange (Fig. 4b,

113 Extended Data Video 16), indicating hyperacetylation differentially affects KMT5C and CBX5

114 demixing in cells. Nevertheless, this modest KMT5C release was associated with a marked

115 accumulation of $\mathrm{H} 4 \mathrm{~K} 20 \mathrm{me} 3$ outside of chromocenters (Fig. 4b), establishing that retention is 
116 essential to the spatial regulation of its enzymatic activity, which otherwise acts promiscuously.

117 Alteration of KMT5C dynamics was also apparent upon induction of DNA damage within

118 chromocenters by laser microirradiation, which is known to induce heterochromatin

119 decompaction and changes in histone post-translational modifications. ${ }^{21}$ Specifically, bisection

120 of chromocenters using laser microirradiation caused loss of KMT5C from the damaged area,

121 creating two lobes that preserved the mobility and retention behavior of the original domain (Fig.

122 4c, Extended Data Videos 17, 18). Unlike the global change observed with TSA treatment, this

123 reflected a locally confined and precise dissolution in response to underlying chromatin state

124 changes over a timescale of seconds. Again, the behavior of KMT5C was markedly different

125 than either $\mathrm{CBX} 5$ or MeCP2, which showed residual localization in the damage zone and rapid

126 mobility (Fig. 4c, d, and Extended Data Videos 19-22). Collectively, these findings indicate that

127 phase separation can tune constitutive heterochromatin protein content and dynamics in response

128 to changes in chromatin state and environmental stimuli.

The CRD provides a minimalist model to decipher how phase separation can support

130 spatial confinement while at the same time maintaining liquid-like behavior. Although lacking

131 many of the typical sequence features of phase separating proteins, ${ }^{3,13}$ the CRD met the criterion

132 of multivalency. Nevertheless, while this normally controls the composition and biophysical

133 properties of phase separated assemblies via low affinity interactions that allow proteins to

134 exchange with their surroundings, ${ }^{3}$ the CRD underwent very limited exchange. In this regard,

135 CRD mutants resembled full-length CBX5 in mobility and partitioning, indicating multiple

136 determinants act in a highly synergistic manner to self-reinforce chromocenter retention. This

137 behavior was independently supported by the response of KMT5C to inhibition of HDACs (Fig.

138 4), which partially diminished retention. For Drosophila HP1a, H3K9me3 recognition is driven 
139 by cation- $\pi$ interactions involving a triad of aromatic residues $\left(\mathrm{Y}^{24}, \mathrm{~W}^{45}\right.$, and $\left.\mathrm{Y}^{48}\right)$ within the

140 chromodomain, ${ }^{15}$ and is necessary for heterochromatin phase separation. ${ }^{2}$ A key difference in the

$141 \mathrm{CRD}$ is the presence of five aromatic residues $\left(\mathrm{W}^{359}, \mathrm{~W}^{384}, \mathrm{~W}^{390}, \mathrm{~W}^{392}\right.$, and $\left.\mathrm{Y}^{396}\right)$ and the

142 predominance of tryptophan, which confers the strongest cation- $\pi$ interactions ${ }^{22}$ and supports

143 more stable binding of trimethyllysine. ${ }^{23}$ Together with multivalency and the capacity for $\pi-\pi$

144 interactions, ${ }^{24}$ the CRD appears to have evolved unique determinants that are optimized to

145 confine KMT5C to constitutive heterochromatin. Moreover, when compared to other phase

146 separating proteins, this activity has been consolidated into a limited number of sequence

147 features that are sufficient to reduce chromocenter surface area and enable exquisite control of

148 protein localization in the aqueous phase.

149 Phase separation can be described in phase diagrams where changes in protein

150 concentration and interaction strength give rise to assemblies with distinct material properties, ${ }^{19}$

151 including gels, glassy solids, and pathological aggregates. ${ }^{13}$ The latter are states where molecules

152 are immobile, retain their relative positions to each other, and do not exchange with their

153 surroundings. ${ }^{19}$ This is clearly not what we have described for KMT5C, which remained in a

154 liquid state despite limited exchange that initially suggested it was immobile. ${ }^{12}$ Although in

155 principle high partitioning to a phase separated compartment has the potential to drive retention, ${ }^{3}$

156 the behavior of $\mathrm{MeCP} 2$ indicates that this feature alone is not sufficient, but also requires a high

157 energy barrier to exit. For KMT5C, this combination allows it to be effectively biocontained

158 within a phase separated 'organelle' and affords tight spatial control of H4K20me3 catalysis.

159 This is notable given it constitutes a minority of the methylated $\mathrm{H} 4 \mathrm{~K} 20$ pool $^{25}$ and is primarily

160 associated with satellite and other distinct repeats. ${ }^{26}$ This control is lost when KMT5C is no

161 longer constrained in demixed assemblies (Fig. 4). Moreover, the opposing behaviors of KMT5C 
162 and MeCP2 indicated that change of a single epigenetic feature can dramatically reprogram the

163 phase separated state with regard to protein composition and dynamics. Of particular relevance

164 to this finding, MeCP2 loss in a mouse model of Rett syndrome leads to H4K20me3 gain in the

165 chromocenter, supporting an antagonistic relationship with KMT5C in vivo at endogenous

166 protein levels. ${ }^{27}$ This principle is also supported by an altered chromocenter proteome in

$167 S u v 39 h 1 / 2$ knockout cells ${ }^{28}$ and the established plasticity of constitutive heterochromatin in

168 development and disease. ${ }^{29}$ The existence of these distinct phase separated states therefore

169 provides a conceptual framework to understand the drivers of normal and pathogenic

170 chromocenter homeostasis. By considering chromatin as a multivalent scaffold, we can decipher

171 how changes in epigenetic features and the mutational status of resident proteins shift the

172 composition and function of phase separated assemblies. 


\section{Methods}

\section{Cell Culture and transfection}

175 Cells were cultured at $37^{\circ} \mathrm{C}$ and $5 \% \mathrm{CO}_{2}$ in a humidified incubator. All cell lines were grown in 176 DMEM containing 10\% FBS. D5 (Suv39h1/2 knockout) and W8 (Suv39h1/2 wild-type) mouse

177 embryonic fibroblast cells lines ${ }^{8}$ were obtained from Dr. Thomas Jenuwein. All other analyses

178 were carried out using the mouse NMuMG breast cell line. Cells were transfected by lipofection

179 using Effectene (Qiagen) 1 day prior to experiments. Expression plasmids were synthesized

180 (www.biomatik.com), obtained from the Addgene repository (www.addgene.org), or previously

181 described $^{30}$ (sequences provided in supplemental material).

$183 \quad$ Live-cell imaging

184 Live cell imaging was carried out using Zeiss Axiovert 200M inverted microscopes attached to

185 either an LSM510 NLO laser scanning system with a $25 \mathrm{~mW}$ argon laser line, a Zeiss LSM 770

186 confocal microscope attached to an Axio Observer Z3 equipped with 405, 488, 561, and $633 \mathrm{~nm}$

187 diode lasers, or a PerkinElmer Ultraview spinning-disk confocal microscope equipped with 405,

188 488, and $561 \mathrm{~nm}$ diode lasers and a FRAP-unit. For all platforms, a 40 x 1.3 NA oil immersion

189 lens was used. Long-term live-cell observations were conducted on the spinning disk microscope

190 at $37^{\circ} \mathrm{C}$ in a humidified atmosphere containing $5 \% \mathrm{CO}_{2}$. In cases were Z-stacks were acquired,

191 spacing was set at $400 \mathrm{~nm}$. Fluorescence recovery after photobleaching was performed on

192 transiently transfected cells using the $488 \mathrm{~nm}$ solid state (spinning disk confocal) or $488 \mathrm{~nm}$

193 argon laser line (LSM 510). Circular (chromocenter) or linear (nucleoplasm) regions were

194 demarcated and subsequently bleached by intense light from the $488 \mathrm{~nm}$ laser. Fluorescence

195 recovery of the bleached regions was quantified over multiple time scales (seconds to minutes). 
196 FRAP data was extracted using Zeiss LSM 5 Zen or ImageJ software by measuring fluorescence

197 intensity of the background, the whole nucleus and the bleached area in each of the recorded

198 time-lapse pictures for a minimum of 30 cells. Normalized relative intensity (including standard

199 deviation) was calculated in Microsoft Excel and plotted using Graphpad Prism software. Laser

200 microirradiation experiments were performed using the spinning disk microscope using the $100 \mathrm{x}$

201 1.4 NA objective lens. Cells were grown in MatTek $35 \mathrm{~mm}$ glass bottom dishes and were

202 sensitized with $1 \mu \mathrm{g} / \mu \mathrm{l}$ Hoechst 20 min prior to the experiment. After calibration of the

203 photokinesis device, a thin horizontal line representing the region to be microirradiated was

204 placed so that it divided the chromocenter into approximately two equal parts. Laser

205 microirradiation was carried out by using $20 \%$ power of the $405 \mathrm{~nm}$ solid state laser and 10

206 iterations. Images were acquired at defined time intervals using laser and filter settings for GFP

207 imaging. Subsequent photobleaching of the non-microirradiated portion of chromocenters was

208 done using 10 iterations at $100 \%$ power of the $488 \mathrm{~nm}$ solid state laser.

\section{Immunofluorescence}

211 Cells grown on adherent coverslips were fixed in 4\% paraformaldehyde in PBS for $10 \mathrm{~min}$,

212 permeabilized in $0.5 \%$ Triton X-100 in PBS for 5min and then incubated with primary

213 antibodies diluted in PBS. After $30 \mathrm{~min}$ at room temperature, the cells were washed once with

$2140.1 \%$ Triton X-100 in PBS for 1 min then rinsed 3 times with PBS prior to addition of secondary

215 antibodies diluted in PBS. Cells were incubated for $30 \mathrm{~min}$ at room temperature, washed with 0.1

$216 \%$ Triton X-100 in PBS for 1 min and rinsed three times with PBS. Coverslips were mounted

217 onto microscope slides with in-house made polyvinyl alcohol mounting media containing 1

$218 \mu \mathrm{g} / \mathrm{mL}$ 4',6-diamidino-2-phenylindole (DAPI). Z-Stacks were obtained on a Zeiss Imager.Z1 
219 equipped with a Photometrics Prime BSI camera and Metamorph software version 7.10.2.240

220 (Molecular Devices, Sunnyvale, CA) using a Zeiss 63 x 1.3 NA oil lens. Step size used was 0.2

$221 \mu \mathrm{m}$. Primary antibodies and dilutions were as follows: $\alpha-H 4 K 20 \mathrm{me} 3$ (Active Motif 39672),

222 1:500; and $\alpha-H 3 K 9 m e 3$ (Active Motif 3916). Secondary antibodies and dilutions were as

223 follows: goat $\alpha$-mouse Alexa-488, 1:500; goat $\alpha$-rabbit Alexa-488 1:500; and goat $\alpha$-mouse

224 Cy3, 1:500.

225

226 Image analysis

227 Images were deconvolved with Huygens Professional version 19.04 (Scientific Volume Imaging,

228 http://svi.nl). Z-stacks were imported into Imaris 9.3 (Oxford Instruments) and cropped to

229 generate 3D images of single nuclei. The Imaris surfaces function was used to encapsulate all

230 chromocentres with a shell and the statistics function was used to measure the volume,

231 sphericity, and total number of chromocenters for output to a Microsoft Excel spreadsheet.

232 Graphical representation of the data was prepared using the PlotsOfDifferences server

233 (https://huygens.science.uva.nl/PlotsOfDifferences/). ${ }^{31}$ For partition coefficients, line scans

234 through nuclei of undeconvolved 3D images were recorded using ImageJ. For each cell, the

235 partition coefficient was calculated by subtracting the background level from the maxima of the

236 brightest chromocenter and dividing it by the background corrected fluorescence intensity of the

237 nucleoplasm. For each protein analyzed, measurements were taken from $\mathrm{n}>10$ nuclei.

\section{Statistical analysis}

240 For partition coefficients, significance was evaluated using the Kruskal-Wallis one-way analysis

241 test and individual comparisons between proteins were done using the Wilcoxon rank sum test. 
242 Statistical significance of sphericity data was evaluated using the embedded stats function within

243 PlotsOfDifferences, which calculates p-values using a randomization test. ${ }^{31}$ 


\section{References}

2451 Larson, A. G. et al. Liquid droplet formation by HP1alpha suggests a role for phase

246 separation in heterochromatin. Nature 547, 236-240, doi:10.1038/nature22822 (2017).

2472 Strom, A. R. et al. Phase separation drives heterochromatin domain formation. Nature

$248 \quad 547,241-245$, doi:10.1038/nature22989 (2017).

2493 Banani, S. F., Lee, H. O., Hyman, A. A. \& Rosen, M. K. Biomolecular condensates:

$250 \quad$ organizers of cellular biochemistry. Nat Rev Mol Cell Biol 18, 285-298,

251 doi:10.1038/nrm.2017.7 (2017).

2524 Alberti, S. The wisdom of crowds: regulating cell function through condensed states of

253 living matter. J Cell Sci 130, 2789-2796, doi:10.1242/jcs.200295 (2017).

2545 Haaf, T. \& Schmid, M. Chromosome topology in mammalian interphase nuclei. Exp Cell

$255 \quad \operatorname{Res} 192,325-332(1991)$.

2566 Erdel, F. \& Rippe, K. Formation of Chromatin Subcompartments by Phase Separation.

257 Biophysical journal 114, 2262-2270, doi:10.1016/j.bpj.2018.03.011 (2018).

2587 Larson, A. G. \& Narlikar, G. J. The Role of Phase Separation in Heterochromatin

259 Formation, Function, and Regulation. Biochemistry 57, 2540-2548,

260 doi:10.1021/acs.biochem.8b00401 (2018).

2618 Peters, A. H. et al. Loss of the Suv39h histone methyltransferases impairs mammalian

262 heterochromatin and genome stability. Cell 107, 323-337 (2001).

2639 Cheutin, T. et al. Maintenance of stable heterochromatin domains by dynamic HP1

$264 \quad$ binding. Science 299, 721-725, doi:10.1126/science.1078572 (2003). 
$26510 \quad$ Muller-Ott, K. et al. Specificity, propagation, and memory of pericentric heterochromatin. Molecular systems biology 10, 746, doi:10.15252/msb.20145377 (2014).

26811 Kumar, A. et al. Analysis of protein domains and Rett syndrome mutations indicate that multiple regions influence chromatin-binding dynamics of the chromatin-associated protein MECP2 in vivo. J Cell Sci 121, 1128-1137, doi:10.1242/jcs.016865 (2008).

$271 \quad 12$ Hahn, M. et al. Suv4-20h2 mediates chromatin compaction and is important for cohesin recruitment to heterochromatin. Genes Dev 27, 859-872, doi:10.1101/gad.210377.112 (2013).

$274 \quad 13$ Shin, Y. \& Brangwynne, C. P. Liquid phase condensation in cell physiology and disease. $275 \quad$ Science 357, doi:10.1126/science.aaf4382 (2017).

27614 Zhu, L. \& Brangwynne, C. P. Nuclear bodies: the emerging biophysics of nucleoplasmic 277 phases. Curr Opin Cell Biol 34, 23-30, doi:10.1016/j.ceb.2015.04.003 (2015).

$27815 \quad$ Jacobs, S. A. \& Khorasanizadeh, S. Structure of HP1 chromodomain bound to a lysine 9methylated histone H3 tail. Science 295, 2080-2083, doi:10.1126/science.1069473 (2002).

28116 Nielsen, P. R. et al. Structure of the HP1 chromodomain bound to histone H3 methylated at lysine 9. Nature 416, 103-107, doi:10.1038/nature722 (2002).

28317 Ohki, I. et al. Solution structure of the methyl-CpG binding domain of human MBD1 in complex with methylated DNA. Cell 105, 487-497 (2001).

28518 Weaver, T. M., Morrison, E. A. \& Musselman, C. A. Reading More than Histones: The doi:10.3390/molecules23102614 (2018). 
28819 Hyman, A. A., Weber, C. A. \& Julicher, F. Liquid-liquid phase separation in biology.

289 Annu Rev Cell Dev Biol 30, 39-58, doi:10.1146/annurev-cellbio-100913-013325 (2014).

29020 Taddei, A., Maison, C., Roche, D. \& Almouzni, G. Reversible disruption of pericentric

291 heterochromatin and centromere function by inhibiting deacetylases. Nat Cell Biol 3,

292 114-120, doi:10.1038/35055010 (2001).

29321 Murray, J. M., Stiff, T. \& Jeggo, P. A. DNA double-strand break repair within

294 heterochromatic regions. Biochemical Society transactions 40, 173-178,

295 doi:10.1042/BST20110631 (2012).

29622 Dougherty, D. A. Cation-pi interactions in chemistry and biology: a new view of

297 benzene, Phe, Tyr, and Trp. Science 271, 163-168, doi:10.1126/science.271.5246.163

298 (1996).

29923 Riemen, A. J. \& Waters, M. L. Design of highly stabilized beta-hairpin peptides through

300 cation-pi interactions of lysine and n-methyllysine with an aromatic pocket. Biochemistry

$301 \quad$ 48, 1525-1531, doi:10.1021/bi801706k (2009).

30224 Vernon, R. M. et al. Pi-Pi contacts are an overlooked protein feature relevant to phase

303 separation. eLife 7, doi:10.7554/eLife.31486 (2018).

30425 Pesavento, J. J., Yang, H., Kelleher, N. L. \& Mizzen, C. A. Certain and progressive

305 methylation of histone H4 at lysine 20 during the cell cycle. Mol Cell Biol 28, 468-486

$306 \quad$ (2008).

30726 Mikkelsen, T. S. et al. Genome-wide maps of chromatin state in pluripotent and lineage-

$308 \quad$ committed cells. Nature 448, 553-560 (2007). 
30927 Linhoff, M. W., Garg, S. K. \& Mandel, G. A high-resolution imaging approach to

310 investigate chromatin architecture in complex tissues. Cell 163, 246-255,

311 doi:10.1016/j.cell.2015.09.002 (2015).

31228 Saksouk, N. et al. Redundant mechanisms to form silent chromatin at pericentromeric

313 regions rely on BEND3 and DNA methylation. Mol Cell 56, 580-594,

314 doi:10.1016/j.molcel.2014.10.001 (2014).

31529 Dejardin, J. Switching between Epigenetic States at Pericentromeric Heterochromatin.

316 Trends Genet 31, 661-672, doi:10.1016/j.tig.2015.09.003 (2015).

31730 Tsang, L. W., Hu, N. \& Underhill, D. A. Comparative analyses of SUV420H1 isoforms

318 and SUV420H2 reveal differences in their cellular localization and effects on myogenic

319 differentiation. PLoS ONE 5, e14447, doi:10.1371/journal.pone.0014447 (2010).

32031 Goedhart, J. PlotsOfDifferences - a web app for the quantitative comparison of unpaired 321 data. bioRxiv, 578575, doi:10.1101/578575 (2019).

32232 Sievers, F. et al. Fast, scalable generation of high-quality protein multiple sequence 323 alignments using Clustal Omega. Molecular systems biology 7, 539,

324 doi:10.1038/msb.2011.75 (2011).

32533 Peng, K. et al. Optimizing long intrinsic disorder predictors with protein evolutionary 326 information. J Bioinform Comput Biol 3, 35-60 (2005).

32734 Drozdetskiy, A., Cole, C., Procter, J. \& Barton, G. J. JPred4: a protein secondary 328 structure prediction server. Nucleic Acids Res 43, W389-394, doi:10.1093/nar/gkv332 329 (2015).

33035 Schotta, G. et al. A silencing pathway to induce H3-K9 and H4-K20 trimethylation at 331 constitutive heterochromatin. Genes Dev 18, 1251-1262 (2004). 


\section{Extended Data}

\section{Videos}

335 Video 1; KMT5C FRAP

336 Video 2; MeCP2 FRAP

337 Video 3; CBX5 FRAP

338 Video 4; Partial KMT5C bleach (doughnut)

339 Video 5; Partial KMT5C bleach (bridge)

340 Video 6; Homo sapiens CRD FRAP

341 Video 7; Mus musculus CRD FRAP

342 Video 8; Cavia porcellus CRD FRAP

343 Video 9; Bubalus bubalis CRD FRAP

344 Video 10; CRDC $^{362} \mathrm{C}^{366}$ FRAP

$345 \quad$ Video 11; $\mathrm{CRDH}^{357} \mathrm{H}^{365}$ FRAP

346 Video 12; $\mathrm{CRDW}^{359} \mathrm{~W}^{390} \mathrm{~W}^{392} \mathrm{Y}^{396}$ FRAP

347 Video 13; $\mathrm{CRDC}^{366} \mathrm{~W}^{390} \mathrm{~W}^{392}$ FRAP

348 Video 14; KMT5C in D5; Suv39h1/2 knockout FRAP

349 Video 15; KMT5C in W8; Suv39h1/2 wild-type FRAP

350 Video 16; KMT5C with TSA FRAP (no TSA control; Video 1)

$351 \quad$ Video 17; KMT5C laser microirradiation

352 Video 18, KMT5C laser microirradiation + FRAP

353 Video 19; MeCP2 laser microirradiation

354 Video 20, MeCP2 laser microirradiation + FRAP

355 Video 21; CBX5 laser microirradiation 
356 Video 22, CBX5 laser microirradiation + FRAP

358 Figures and Tables

359 Table 1; Spreadsheet 1; PCH PONDR excel; additional species disorder and physicochemical

360 data for KMT5C, MeCP2, CBX5, and CRD

361 Figure 1; Sphericity data for (a) KMT5C, MeCP2, CBX5, (b) $H s, C p, B b$, and $C p$ CRDs, and (c)

362 wild-type and mutant CRDs.

363 Figure 2; MeCP2 (a) localization and (b) mobility in D5 (Suv39h1/2 knockout) and W8

364 (Suv39h1/2 wild-type) cells 


\section{Acknowledgements}

366 The authors acknowledge funding support from the Canadian Breast Cancer Foundation

367 (D.A.U., grant no. 300073), Cancer Research Society (D.A.U. and M.J.H., grant no. CRSDI

3682018 OG 23446), and Canadian Institutes of Health Research (M.J.H., grant no. PJT-148753).

369 The authors thank Dr. Xuejun Sun and Gerry Barron of the Cross Cancer Institute Cell Imaging

370 Facility for support.

371

372 Author Contributions

373 D.A.U. and M.J.H. conceived the project. D.A.U., M.J.H, and H.S. designed all experiments and

374 interpreted data. K.M. made the initial observation of KMT5C mobility and performed FRAP

375 and fluorescence imaging experiments. H.S. performed live-cell imaging, laser micro-irradiation,

376 and partition coefficient analyses. D.A.U. carried out sequence analysis and expression plasmid

377 design. D.A.U. wrote the manuscript with contributions from M.J.H. and H.S. D.A.U. prepared

378 figures with contributions from H.S. and K.M.

380 Containing data deposition statement

$381 \quad \mathrm{~N} / \mathrm{A}$

382

\section{Competing interests}

384 The authors declare no competing interests. 


\section{Corresponding author line}

387 D. A. Underhill Department of Oncology, 2328 Cross Cancer Institute, 11560 University

388 Avenue, Edmonton, Alberta, Canada, T6G 1Z2. Phone: (780) 432-8903, Fax: (780) 432-8892,

389 E-mail: alan.underhill@ualberta.ca 


\section{Figure 1. KMT5C is mobile within chromocenters but undergoes limited nucleoplasmic}

391 exchange. a, FRAP curves for KMT5C, MeCP2, and CBX5-mEmerald fusion proteins in mouse

392 NMuMG breast cancer cells ( $n=30$ ) (see Methods). Partial (red) and full (blue) fluorescence

393 recovery curves (filled circles represent mean fluorescence intensity, while vertical lines indicate

394 standard deviation. b, Time-lapse series of full and partial KMT5C-mEmerald photobleaching

395 (Extended Data Movie 4). Insets use a 16-color intensity map to depict fluorescence recovery

396 upon partial or full bleaching from 0.3 to 60 s. The second partial panel depicts an independent

397 recovery event involving a chromatin bridge between two chromocenters (Extended Data Movie

398 5), indicating they form a contiguous phase separated environment. c, Schematic representation

399 of KMT5C (green) movement (arrows) in each of the full and partial bleach (translucent circles)

400 time series from panel $\boldsymbol{b}$. d, Partition images (16-color intensity map) for KMT5C, CBX5 and

401 MePC2. e, Scatter plots display corresponding partition coefficients for KMT5B ( $n=26), \mathrm{MeCP} 2$

$402(n=30)$, and CBX5 $(n=30)$ (see Methods). 

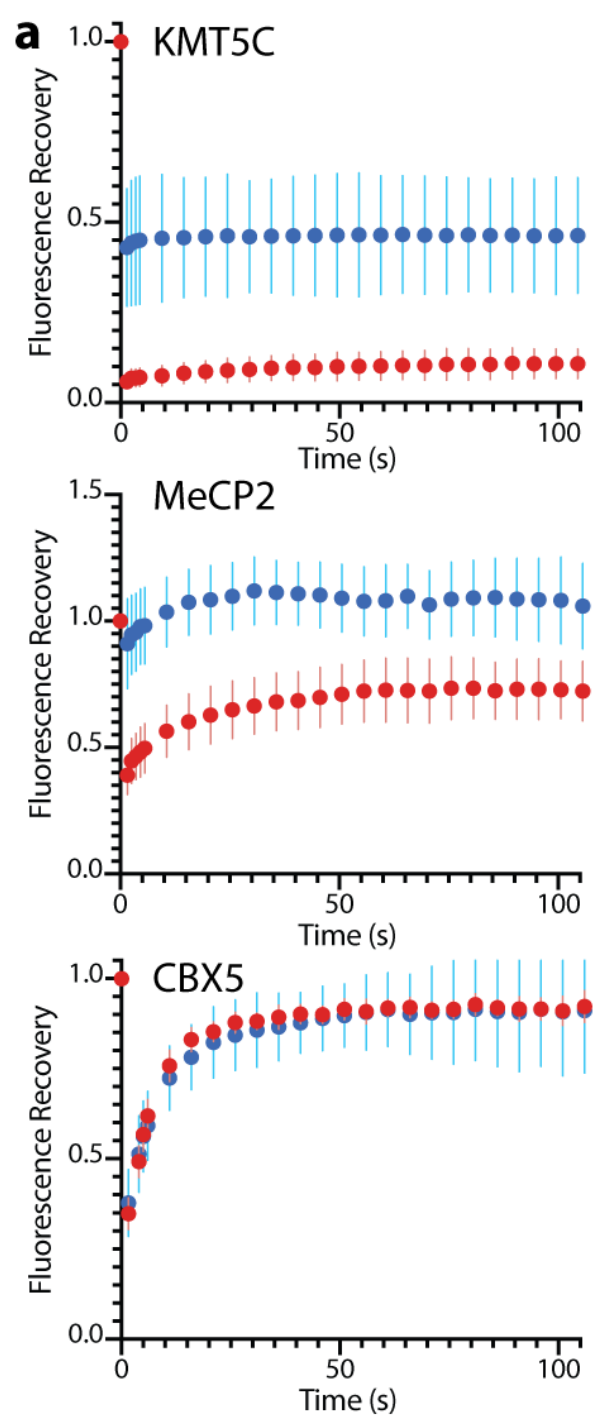

b
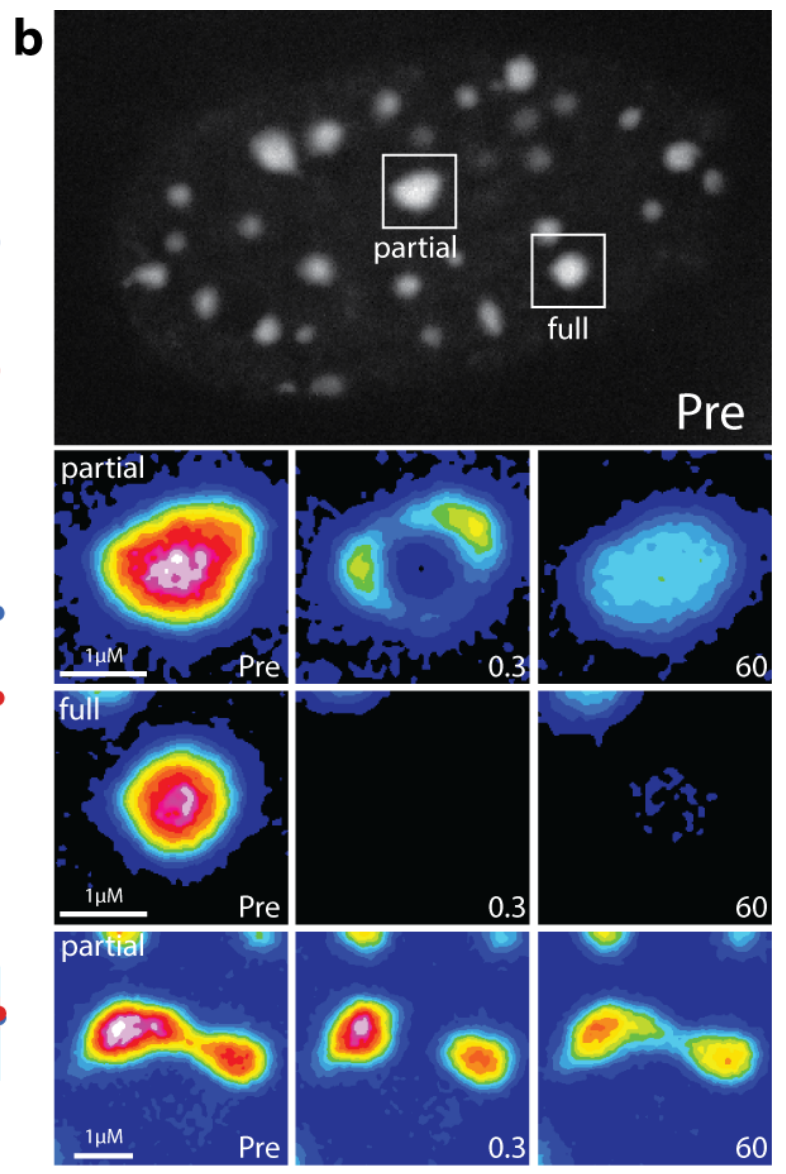

C

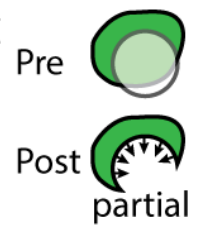

d
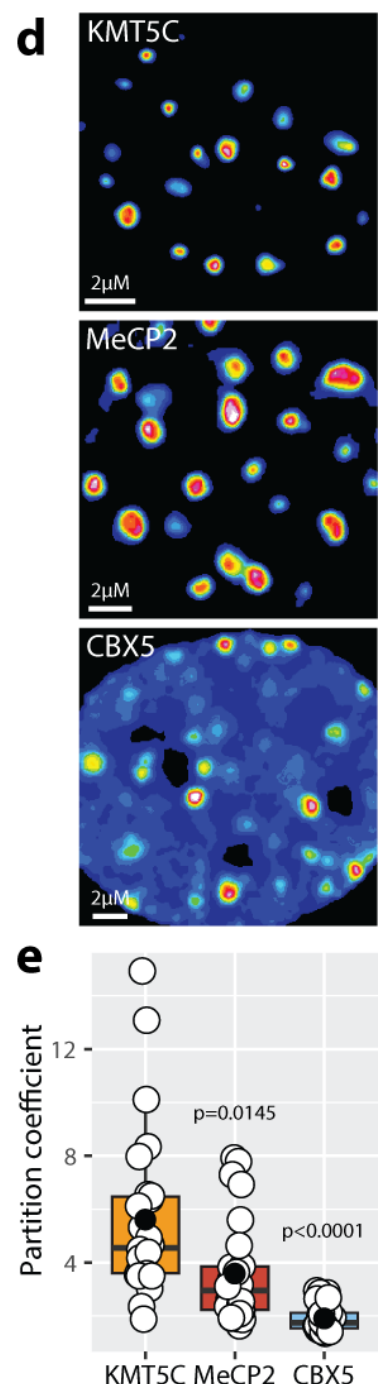
Figure 2. The chromocenter retention domain constrains KMT5C to individual

chromocenters. a, Disorder plots (PONDR) and charge heatmaps for KMT5C, CBX5, and MeCP2 orthologs from Homo Sapiens (Hs), Mus musculus (Mm), Bubalus bubalis (Bb), and

408 Cavia porcellus $(C p)$. For reference, schematics illustrate the distribution of annotated domains

409 in each protein ( $\mathrm{Su}(\mathrm{var}) 3-9$, Enhancer-of-zeste and Trithorax (SET); RNA-Binding Domain

410 (RBD); Chromocenter Retention Domain; Methyl-CpG Binding Domain (MBD);

411 Chromodomain (CD); Hinge domain (H); and Chromoshadow Domain (CD)). Input sequences

412 were aligned using Clustal Omega ${ }^{32}$ together with manual removal of gaps and then analyzed for

413 disorder using PONDR ${ }^{33}$ and charge properties using EMBOSS. Average disorder percentage

414 and score are indicated to the right of the primary structure schematic, and disordered segments

415 are shown as thick horizontal lines. b, Multispecies sequence alignment of the CRD is shown in

416 JPred $^{34}$ format. Residues absolutely conserved in mammals are indicated by a filled circle, while

417 those exhibiting 90\% conservation are noted by open circles. Amino acids selected for

418 mutagenesis are numbered. c, Disorder plots and charge heatmaps are shown for the CRD

419 (species and details are as described in panel $a$ ). d, Scatter plots of isoelectric point, disorder

420 score, disorder \%, and disorder length for CBX5, CRD, KMT5C, and MeCP2 across 20

421 representative mammalian species (Extended Data Table 1). e, Partition images (16-color

422 intensity map) for the CRD from $H s, M m, C p$, and $B b$ indicate that all effectively partition to

423 chromocenters. f, Partition coefficient graph for $H s(n=39), M m(n=30), C p(n=23)$, and $B b$

$424(n=26)$ CRDs demonstrate high chromocenter partitioning. Full-length KMT5C is shown for

425 reference (faded). g, FRAP analyses of $H s, M m, C p$, and $B b$ CRD domains (Extended Data

426 Movies 6-9) support intra-chromocenter mobility with reduced nucleoplasmic exchange $(n=30)$. 

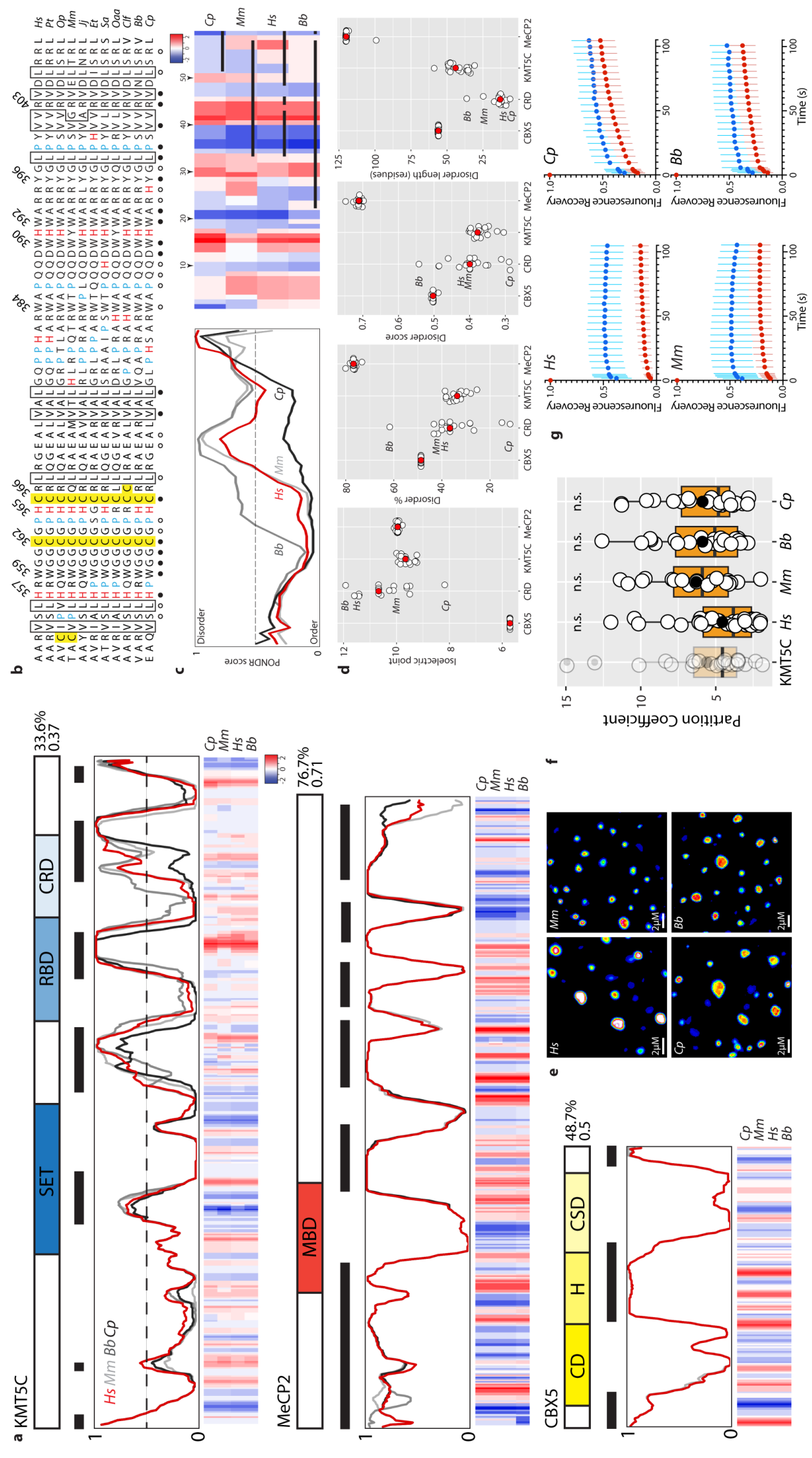
429 Figure 3. The CRD comprises multiple determinants that cooperate to drive

430 heterochromatin phase separation. a, FRAP analysis of CRD mutants that target conserved

431 sequence features $\left(\mathrm{C}^{362} \mathrm{C}^{366}, \mathrm{H}^{357} \mathrm{H}^{365}\right.$, and $\left.\mathrm{W}^{359} \mathrm{~W}^{390} \mathrm{~W}^{392} \mathrm{Y}^{396}\right)$ or combinations thereof

$432\left(\mathrm{C}^{366} \mathrm{~W}^{390} \mathrm{~W}^{392}\right)(n=30)$. Wild-type KMT5C (faded) is shown in the upper left panel for reference

433 (Extended Data movies 10-13). While the recovery profiles are similar for the $\mathrm{C}^{362} \mathrm{C}^{366}$ and

$434 \quad \mathrm{H}^{357} \mathrm{H}^{365}$ mutants, they differed for the $\mathrm{W}^{359} \mathrm{~W}^{390} \mathrm{~W}^{392} \mathrm{Y}^{396}$ and mutants, suggesting they affect

435 distinct interactions. b, Partition images (16-color intensity map) for CRD mutants. c, Partition

436 coefficients for CRD mutants (WT $(n=39)$, Trp $(n=18)$, His $(n=17)$, and Cys $(n=20))$. d,

437 Schematic summary illustrates that mutants continue to localize to the chromocenter, but now

438 readily exchange with the cytoplasmic pool. e, Sphericity analysis of the wild-type $(n=990)$ and

439 mutant $(\operatorname{Trp}(n=437)$, His $(n=1834)$, and Cys $(n=1054)$ CRDs (see Methods). 
a
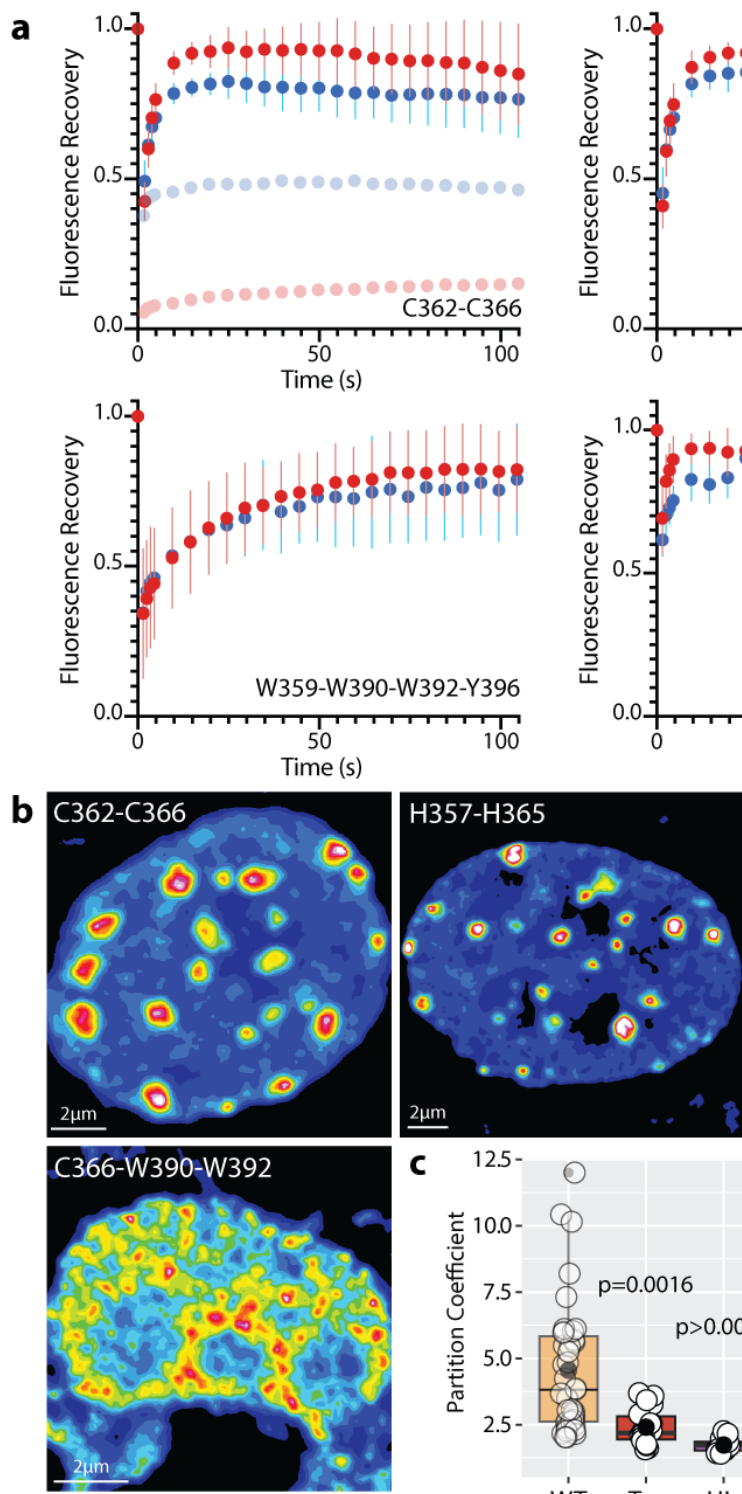

C $\quad 12.5$

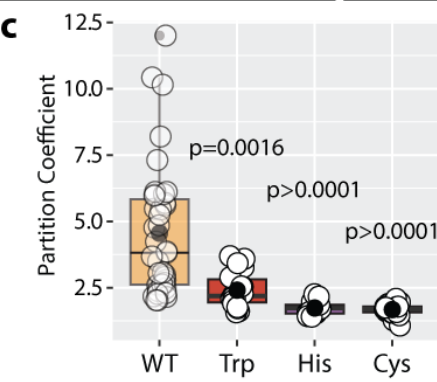

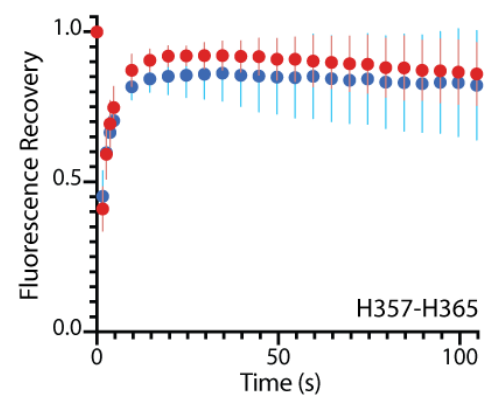
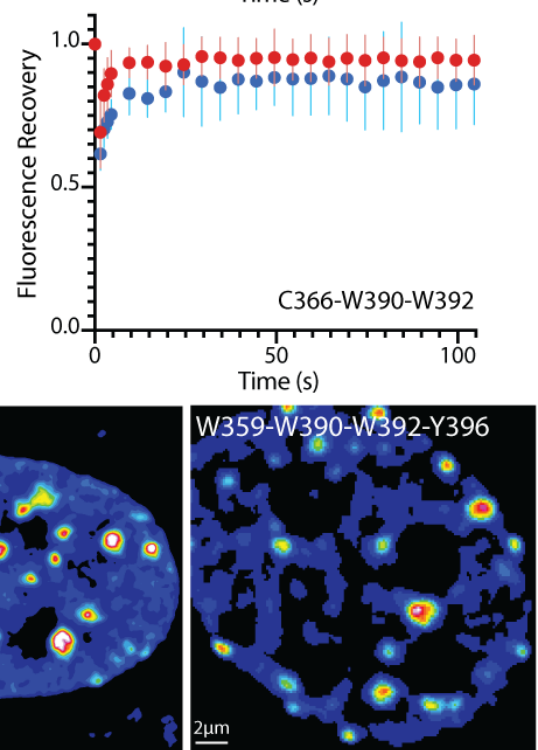

d

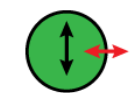

KMT5C, CRD

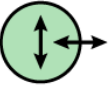

Cys, His, Trp

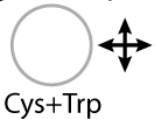

e

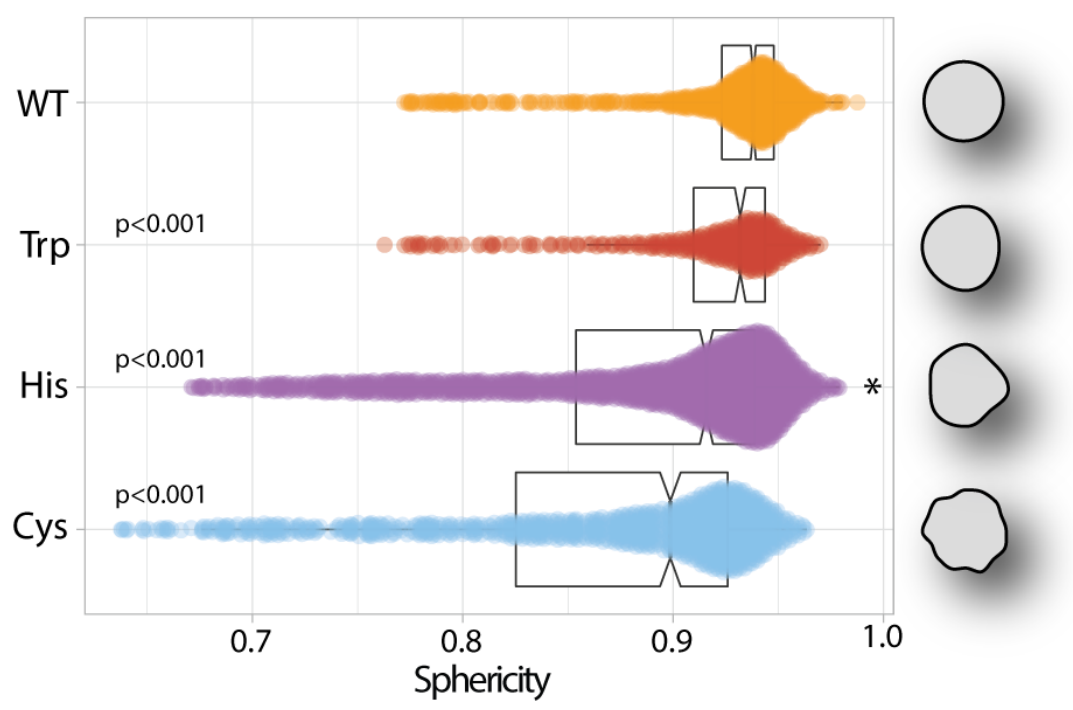


443 Figure 4. KMT5C phase separation is rapidly reversible and responsive to underlying

444 chromatin state. a, KMT5C localization and mobility in Suv39h1/2 knockout cells. Top row,

445 KMT5C localization is dependent on H3K9me3 placement by SUV39H1/2 ${ }^{35}$ which leads to

446 redistribution of H4K20me3 (middle row) and marked in increase in KMT5C mobility in

447 Suv39h1/2 knockout cells when assessed by FRAP (bottom row and Extended Data Videos 14,

448 15) $(n=30)$. b, The histone deacetylase inhibitor trichostatin A (TSA) alters KMT5C localization

449 and mobility, and leads to H4K20me3 redistribution (treatment was for $16-24 \mathrm{hrs}$ at $100 \mathrm{nM}$ ).

450 Upper row, KMT5C localization is only moderately affected by TSA treatment, but H4K20me3

451 undergoes dramatic redistribution (middle row) that coincides with increased KMT5C

452 nucleoplasmic exchange in FRAP (bottom row and Extended Data Video 16) $(n=30)$. In $a$ and $b$,

453 the schematic indicates H4K20me3 is no longer confined to the chromocenter under either

454 condition, but that Suv39h1/2 knockout had a larger effect on KMT5C localization, despite

455 similar increases in mobility with TSA treatment. c, Laser micro-irradiation of KMT5C, MeCP2,

456 and CBX5 differentially modulates their phase separation (Extended Data Videos 17-22). For

457 each protein, the top row represents a time series that includes pre-damage (Pre), immediately

458 following damage (4s), and at 60s (area targeted by micro-IR is indicated by facing arrows). The

459 bottom row depicts a FRAP time series where a portion of the damaged chromocenter (arrows in

460 Pre-bleach) is photobleached (red arrow) and recovery is monitored at 4s and either 60s

461 (KMT5C) or 20s (MeCP2 and CBX5). Maximum image projections (MIP) are included to

462 highlight the area of decondensed heterochromatin following damage induction and the location

463 of the bleaching zone for mEmerald fusions of $\mathrm{KMT} 5 \mathrm{C}, \mathrm{MeCP} 2$, and $\mathrm{CBX}$. d, Comparison of

464 pre and post irradiation images for KMT5C, MeCP2 and CBX5. While MeCP2 and CBX5

465 behaved similarly and underwent only transient or no decrease in intensity over the damaged 
466 area, KMT5C rapidly exited and accumulated in the two non-damaged lobes. e, Schematic

467 summary of laser microirradiation and FRAP analyses. The left panel summarizes the

468 experimental strategy, which involves using a laser to induce chromocenter DNA damage and

469 then characterize the behavior of the exogenously expressed fusion protein, as well as its

470 capacity to recover fluorescence following photobleaching. The DAPI series was used to monitor

471 heterochromatin decompaction following damage, while the response of the mEmerald fusion

472 protein was visualized over time at which point the lower portion of chromocenter was bleached

473 in order to determine protein mobility. In both contexts, KMT5C behaved markedly different

474 than MeCP2 and CBX5. It neither persisted in the damaged area nor exhibited fluorescence

475 recovery.

476 

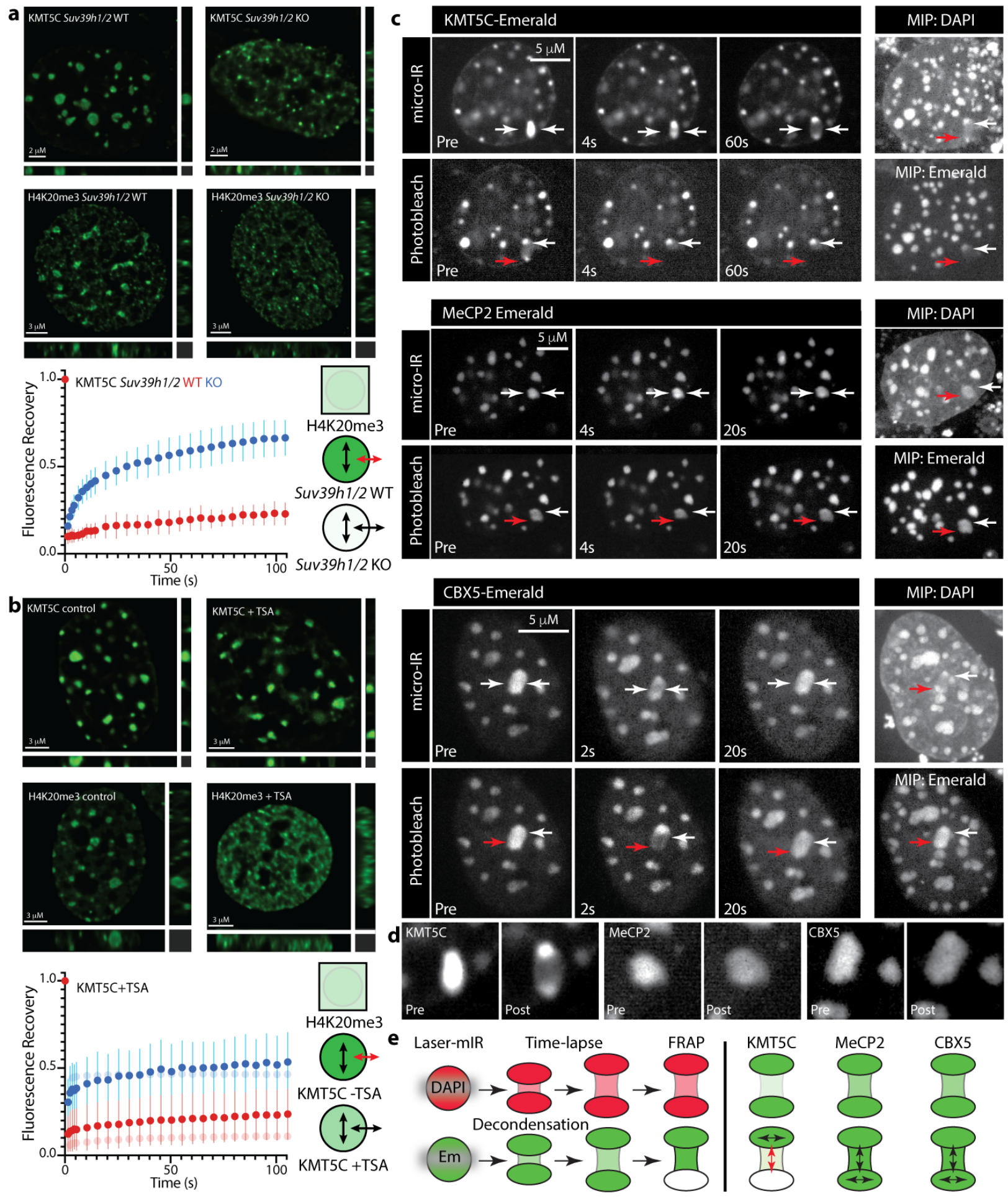


\section{Extended Data Figures and Legends}

479 Figure 1. Chromocenter sphericity analysis of KMT5, MeCP2, CBX5, and all CRD

480 derivatives. a, Comparison of chromocenter sphericity of full-length KMT5C ( $n=775), \mathrm{MeCP} 2$

$481 \quad(n=641)$, and CBX5 $(n=644)$ indicates KMT5C supports significantly higher sphericity. b,

482 Comparison of chromocenter sphericity generated by CRDs from Homo sapiens (Hs; $n=990)$,

483 Mus musculus (Mm; $n=421)$, Bubalus bubalis $(B b ; n=574)$, and Cavia porcellus $(C p ; n=982)$.

484 All CRDs support efficient sphericity, although this is significantly higher for $M m, B b$, and $C p$

485 when compared to $H s$, which also exhibited reduced chromocenter portioning (n.s.) (manuscript

486 Fig. 2f) 2. Nevertheless, the human CRD supports significantly higher sphericity than MeCP2

$487(\mathrm{p}<0.001)$ and CBX5 ( $<<0.001)$, or CRD mutants (panel c). $\mathrm{c}$, Comparison of sphericity for wild-

488 type $(H s)$ and mutant CRDs (as in manuscript Fig.3e, with inclusion of difference plot). All

489 proteins were assessed in murine NMuMG breast cancer cells (see Methods). 
bioRxiv preprint doi: https://doi.org/10.1101/776625; this version posted September 20, 2019. The copyright holder for this preprint (which was not certified by peer review) is the author/funder. All rights reserved. No reuse allowed without permission.
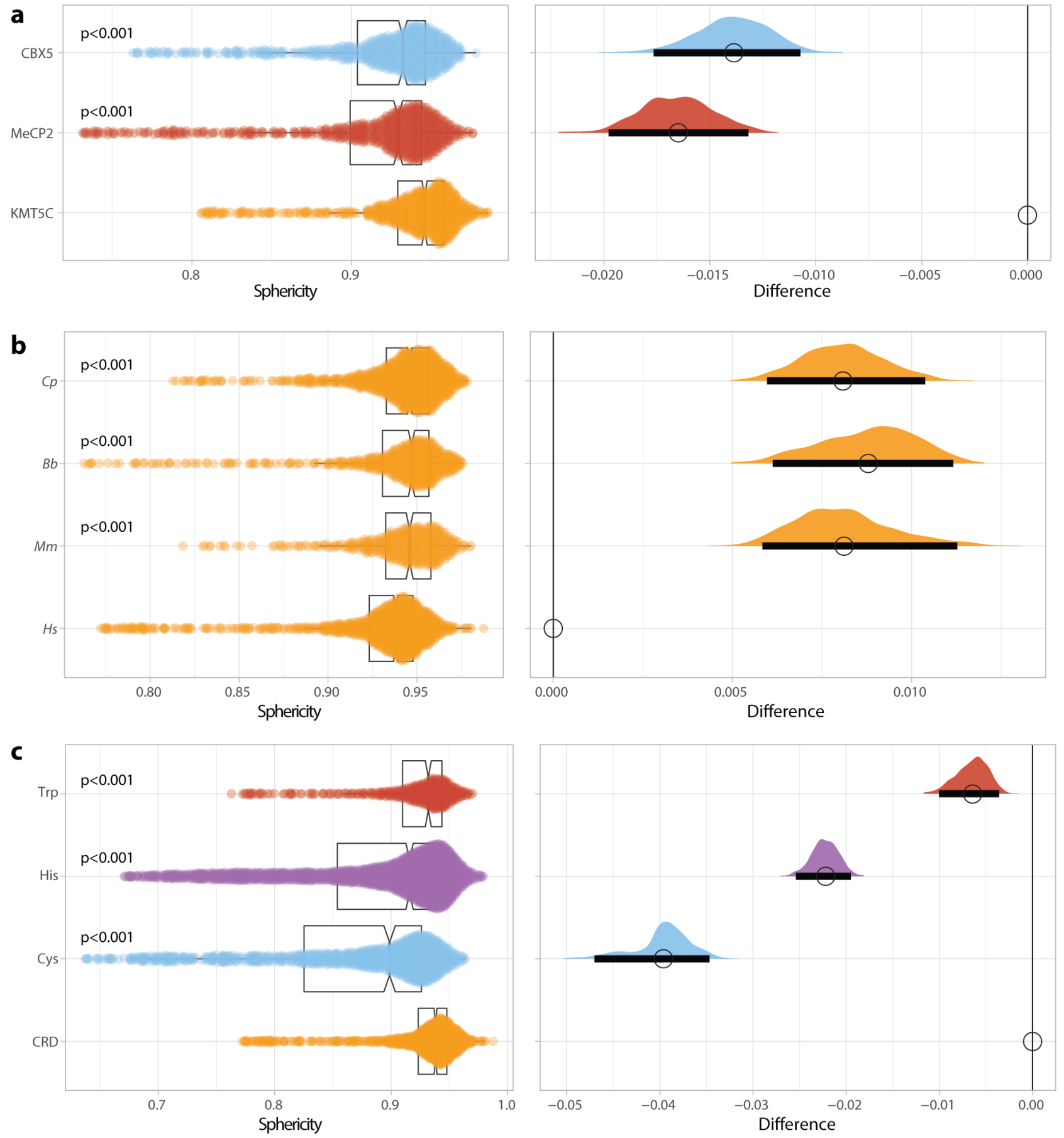
492 Figure 2. Localization and FRAP of MeCP2 in Suv39h1/2 wild-type and knockout cells. a,

493 MeCP2-GFP displays efficient chromocenter localization in both Suv39h1/2 wild-type and

494 knockout mouse embryonic fibroblasts. b, FRAP analysis of MeCP2-GFP reveals a decrease in

495 mobility in Suv39h1/2 knockout cells.

496 

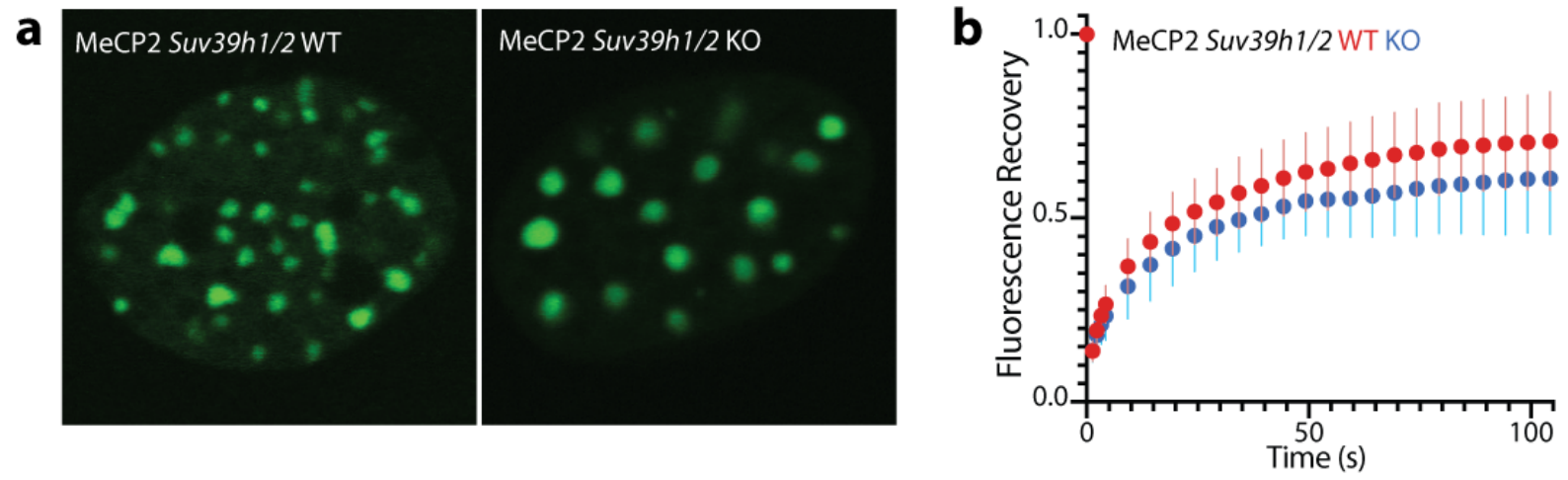